\title{
A GENERALIZED THERMOELASTIC DIFFUSION PROBLEM FOR AN INFINITELY LONG SOLID CYLINDER
}

\author{
MONCEF AOUADI
}

Received 20 June 2005; Revised 8 January 2006; Accepted 12 March 2006

The theory of generalized thermoelastic diffusion, based on the theory of Lord and Shulman, is used to study the thermoelastic-diffusion interactions in an infinitely long solid cylinder subjected to a thermal shock on its surface which is in contact with a permeating substance. By means of the Laplace transform and numerical Laplace inversion the problem is solved. Numerical results predict finite speeds of propagation for thermoelastic and diffusive waves and the presence of a tensile stress region close to the cylinder surface. The problem of generalized thermoelasticity has been reduced as a special case of our problem.

Copyright (c) 2006 Hindawi Publishing Corporation. All rights reserved.

\section{Introduction}

Thermodiffusion in an elastic solid is due to coupling of the fields of temperature, mass diffusion and that of strain. Heat and mass exchange with the environment during thermodiffusion in an elastic solid. These days, oil companies are interested in the process of thermodiffusion for more efficient extraction of oil from oil deposits. Diffusion can be defined as the random walk of an ensemble of particles from regions of high concentration to regions of lower concentration. In integrated circuit fabrication, diffusion is used to introduce "dopants" in controlled amounts into the semiconductor substrate. In particular, diffusion is used to form the base and emitter in bipolar transistors, form integrated resistors, form the source/drain regions in MOS transistors, and dope polysilicon gates in MOS transistors. In most of theses applications, the concentration is calculated using what is known as Fick's law. This is a simple law that does not take into consideration the mutual interaction between the introduced substance and the medium into which it is introduced or the effect of the temperature on this interaction.

Nowacki [7-10] developed the theory of thermoelastic diffusion. In this theory, the coupled thermoelastic model is used. This implies infinite speeds of propagation of thermoelastic waves. In the coupled theory of thermoelasticity the velocity of heat propagation is assumed to be infinitely large. To eliminate this paradox a generalized thermoelasticity theory has been developed subsequently. The development of this theory was 
accelerated by the advent of the second sound effects observed experimentally in materials at a very low temperature. In heat transfer problems involving very short time intervals and/or very high heat fluxes, it has been revealed that the inclusion of the second sound effects to the original theory yields results which are realistic and very much different from those obtained with classical theory of elasticity. The first theory was developed by Lord and Shulman [6]. In this theory a modified law of heat conduction including both the heat flux and its time derivative replaces the conventional Fourier law. The heat equation associated with this a hyperbolic one and, hence, automatically eliminates the paradox of infinite speeds of propagation inherent in the coupled theory of thermoelasticity.

Recently Sherief et al. [15] developed the theory of generalized thermoelastic diffusion with one relaxation time, which allows the finite speeds of propagation of waves. This development provides a chance to study the wave propagation in such an interesting media. Sherief and Saleh [16] investigated the problem of a thermoelastic half-space in the context of the theory of generalized thermoelastic diffusion with one relaxation time. Singh [17] discussed the reflection phenomena of waves from free surface of an elastic solid with generalized thermodiffusion. Aouadi studied in [2] the generalized thermoelastic diffusion problem with variable electrical and thermal conductivity.

Great attention has been devoted to the study of thermoelastic interactions of an infinitely long electrically and thermally conducting annular cylinder. In the context of LordShulman theory, Sherief and Ezzat [14], Santawa and Roychoudhuri [12], Sherief [13], and He et al. [4] studied magneto-thermoelastic interactions of an infinitely long cylinder when the surface of the cylinder is subjected to different boundary conditions. Recently, Aouadi [1] investigated discontinuities of solutions in an axisymmetric cylinder under three thermoelastic theories.

The above investigations are concerned with elastic solid without considering the diffusion phenomena. This paper is devoted to the study of the interaction between the processes of elasticity, heat, and diffusion in an infinitely long solid cylinder in the context of the theory of generalized thermoelastic diffusion with one relaxation time. Numerical results predict finite speeds of propagation for thermoelastic and diffusive waves and the presence of a tensile stress region close to the cylinder surface.

\section{Formulation of the problem}

Following, Sherief et al. $[15,16]$ are the governing equations for an isotropic, homogenous elastic solid with generalized thermodiffusion at constant temperature $T_{0}$ in the absence of body forces:

(i) the constitutive equations:

$$
\begin{gathered}
\sigma_{i j}=2 \mu e_{i j}+\delta_{i j}\left[\lambda e_{k k}-\beta_{1}\left(T-T_{0}\right)-\beta_{2} C\right], \\
P=-\beta_{2} e_{k k}+b C-c\left(T-T_{0}\right)
\end{gathered}
$$

(ii) the equation of motion in the absence of external body forces:

$$
\sigma_{i j, j}=\rho \frac{\partial^{2} u_{i}}{\partial t^{2}}
$$


(iii) the equation of heat conduction:

$$
\left(\frac{\partial}{\partial t}+\tau_{0} \frac{\partial^{2}}{\partial t^{2}}\right)\left(\rho c_{E} T+T_{0} \beta_{1} e_{k k}+c T_{0} C\right)=k T_{, i i}
$$

(iv) the equation of mass diffusion:

$$
D \beta_{2} e_{k k, i i}+D c T_{, i i}+\left(\frac{\partial}{\partial t}+\tau \frac{\partial^{2}}{\partial t^{2}}\right) C=D b C_{, i i}
$$

where $\beta_{1}=(3 \lambda+2 \mu) \alpha_{t}$ and $\beta_{2}=(3 \lambda+2 \mu) \alpha_{c}, \alpha_{t}$ and $\alpha_{c}$ are, respectively, the coefficients of linear thermal and diffusion expansions, $\lambda$ and $\mu$ are Lamé's constants. $T$ is the absolute temperature of the medium, $T_{0}$ is the reference uniform temperature of the body chosen such that $\left|\left(T-T_{0}\right) / T_{0}\right| \ll 1, k$ is the coefficient of thermal conductivity, $c_{E}$ is the specific heat at constant strain. $\sigma_{i j}$ are the components of the stress tensor, $e_{i j}$ are the components of the strain tensor, $P$ is the chemical potential, $C$ is the concentration of the diffusive material in the elastic body, $D$ is the diffusion coefficient, ' $c$ ' is a measure of thermodiffusion effect, ' $b$ ' is a measure of diffusive effect, and $\rho$ is the mass density. $\tau_{0}$ is the thermal relaxation time, which will ensure that the heat conduction equation will predict finite speeds of heat propagation, and $\tau$ is the diffusion relaxation time, which will ensure that the equation satisfied by the concentration $C$ will also predict finite speeds of propagation of matter from one medium to the other.

As the density is assumed to be a constant, the equation of motion (2.3) for this theory remain the same as the one for the thermoelastic theory (see [2, 15-17]). The remaining governing equations, namely, the equation of heat conduction and constitutive relations are different from those for the thermoelastic theory by including mass diffusion terms. The generalized thermoelastic problem can be reduced as a special case of the thermoelastic diffusion theory.

We consider an infinitely long isotropic solid cylinder. $(r, \varphi, z)$ are taken as the cylindrical coordinates with $z$-axis along the axial direction of the cylinder. The surface of the cylinder which in contact with a permeating substance (such a gas) is subjected in a time $t=0$ to a thermal shock. We note that due to symmetries of the cylinder, the only nonvanishing displacement component is the radial one $u_{r}=u(r, t)$. The strain tensor has the following components:

$$
e_{r r}=\frac{\partial u}{\partial r}, \quad e_{\varphi \varphi}=\frac{u}{r}, \quad e_{r \varphi}=e_{\varphi z}=e_{r z}=e_{z z}=0
$$

The cubic dilatation $e$ is thus given by

$$
e=\frac{\partial u}{\partial r}+\frac{u}{r}=\frac{1}{r} \frac{\partial(r u)}{\partial r} .
$$


4 A generalized thermoelastic diffusion problem

The stress-strain relations have the form

$$
\begin{gathered}
\sigma_{r r}=2 \mu \frac{\partial u}{\partial r}+\lambda e-\beta_{1}\left(T-T_{0}\right)-\beta_{2} C, \\
\sigma_{\varphi \varphi}=2 \mu \frac{u}{r}+\lambda e-\beta_{1}\left(T-T_{0}\right)-\beta_{2} C, \\
\sigma_{z z}=\lambda e-\beta_{1}\left(T-T_{0}\right)-\beta_{2} C .
\end{gathered}
$$

The motion (2.3) then reduces to

$$
\sigma_{r r, r}+\frac{\sigma_{r r}-\sigma_{\varphi \varphi}}{r}=\rho \frac{\partial^{2} u}{\partial t^{2}}
$$

Thus from (2.8)-(2.9), we obtain

$$
(\lambda+2 \mu) \frac{\partial e}{\partial r}-\beta_{1} \frac{\partial T}{\partial r}-\beta_{2} \frac{\partial C}{\partial r}=\rho \frac{\partial^{2} u}{\partial t^{2}}
$$

Applying the operator $(1 / r)(\partial / \partial r)(r)$ to both sides of the above equation, we obtain

$$
(\lambda+2 \mu) \nabla^{2} e-\beta_{1} \nabla^{2} T-\beta_{2} \nabla^{2} C=\rho \frac{\partial^{2} e}{\partial t^{2}},
$$

where the Laplacian operator $\nabla^{2}$ is given by

$$
\nabla^{2}=\frac{\partial^{2}}{\partial r^{2}}+\frac{1}{r} \frac{\partial}{\partial r}
$$

The generalized equation of heat conduction (2.4) reduces to

$$
k \nabla^{2} T=\left(\frac{\partial}{\partial t}+\tau_{0} \frac{\partial^{2}}{\partial t^{2}}\right)\left(\rho c_{E} T+T_{0} \beta_{1} e+c T_{0} C\right) .
$$

The diffusion equation (2.5) takes the form

$$
D b \nabla^{2} C=D \beta_{2} e+D c \nabla^{2} T+\left(\frac{\partial}{\partial t}+\tau \frac{\partial^{2}}{\partial t^{2}}\right) C
$$

Now we introduce the following nondimensional variables:

$$
\begin{gathered}
r^{*}=c_{1} \eta_{0} r, \quad u^{*}=c_{1} \eta_{0} u, \quad \theta=\frac{\beta_{1}\left(T-T_{0}\right)}{\lambda+2 \mu}, \quad C^{*}=\frac{\beta_{2} C}{\lambda+2 \mu}, \\
\sigma_{i j}^{*}=\frac{\sigma_{i j}}{\lambda+2 \mu}, \quad P^{*}=\frac{P}{\beta_{2}}, \quad t^{*}=c_{1}^{2} \eta_{0} t, \quad \tau_{0}^{*}=c_{1}^{2} \eta_{0} \tau_{0}, \quad \tau^{*}=c_{1}^{2} \eta_{0} \tau,
\end{gathered}
$$


where $c_{1}^{2}=(\lambda+2 \mu) / \rho, \eta_{0}=\rho c_{1} / k$. In terms of these nondimensional variables, (2.11)(2.14) take the following form (dropping the asterisks for convenience):

$$
\begin{gathered}
\frac{\partial^{2} e}{\partial t^{2}}=\nabla^{2} e-\nabla^{2} \theta-\nabla^{2} C, \\
\nabla^{2} \theta=\left(\frac{\partial}{\partial t}+\tau_{0} \frac{\partial^{2}}{\partial t^{2}}\right)\left(\theta+\varepsilon e+\varepsilon \alpha_{1} C\right), \\
\alpha_{3} \nabla^{2} C=\alpha_{2}\left(\frac{\partial}{\partial t}+\tau \frac{\partial^{2}}{\partial t^{2}}\right) C+\nabla^{2} e+\alpha_{1} \nabla^{2} \theta .
\end{gathered}
$$

The constitutive equations reduce to

$$
\begin{gathered}
\sigma_{r r}=e-\frac{2}{\beta^{2}} \frac{u}{r}-\theta-C, \\
\sigma_{\varphi \varphi}=-\frac{2}{\beta^{2}} \frac{\partial u}{\partial r}+e-\theta-C, \\
\sigma_{z z}=\left(1-\frac{2}{\beta^{2}}\right) e-\theta-C, \\
P=\alpha_{3} C-e-\alpha_{1} \theta,
\end{gathered}
$$

where

$$
\varepsilon=\frac{T_{0} \beta_{1}^{2}}{\rho^{2} c_{E} c_{1}^{2}}, \quad \alpha_{1}=\frac{a \rho c_{1}^{2}}{\beta_{1} \beta_{2}}, \quad \alpha_{2}=\frac{\mu}{D \eta}, \quad \alpha_{3}=\frac{b \rho c_{1}^{2}}{\beta_{2}^{2}}, \quad \beta^{2}=\frac{\lambda+2 \mu}{\mu} .
$$

In order to solve the problem, the surface of the cylinder is taken to be traction free and subjected to a time dependent thermal shock. The chemical potential is also assumed to be a known function of time on the surface of the cylinder. The homogeneous initial conditions are supplemented by the following boundary conditions.

(1) The surface of the cylinder is traction free, this gives

$$
\sigma_{r r}(a, t)=0
$$

This means that the surface of the cylinder is traction free, that is, nonmechanical loads on the surface.

(2) The thermal boundary condition is that the surface of the cylinder is subjected to a thermal shock:

$$
\theta(a, t)=\theta_{0} H(t)
$$

where $H(t)$ is the Heaviside unit step function. This means that the heat is applied on the surface of the cylinder to keep it at a constant temperature $\theta_{0}$ for $t \geq 0$.

(3) The chemical potential is also assumed to be a known function of time at the surface of the cylinder:

$$
P(a, t)=P_{0} H(t)
$$


6 A generalized thermoelastic diffusion problem

\section{Solution in the Laplace transform domain}

Introducing the Laplace transform defined by the formula

$$
\bar{f}(x, s)=\mathfrak{E}(f(x, t))=\int_{0}^{\infty} f(x, t) e^{-s t} d t, \quad \mathfrak{R} e(s)>0,
$$

into (2.16) and using the homogeneous initial conditions, we obtain

$$
\begin{gathered}
s^{2} e=\nabla^{2} e-\nabla^{2} \theta-\nabla^{2} C, \\
\nabla^{2} \theta=s\left(1+\tau_{0} s\right)\left(\theta+\varepsilon e+\varepsilon \alpha_{1} C\right), \\
\alpha_{3} \nabla^{2} C=\alpha_{2} s(1+\tau s) C+\nabla^{2} e+\alpha_{1} \nabla^{2} \theta .
\end{gathered}
$$

The above system of equations can be written in the form

$$
\left(\nabla^{6}-a_{1} \nabla^{4}+a_{2} \nabla^{2}-a_{3}\right)(\bar{e}, \bar{\theta}, \bar{C})=0,
$$

where

$$
\begin{gathered}
a_{1}=\frac{s}{\alpha_{3}-1}\left[\alpha_{3} s+\left(1+\tau_{0} s\right)\left[\alpha_{1} \varepsilon\left(2+\alpha_{1}\right)+\alpha_{3}(\varepsilon+1)-1\right]+(1+\tau s) \alpha_{2}\right] \\
a_{2}=\frac{s^{2}}{\alpha_{3}-1}\left[\left(1+\tau_{0} s\right)\left(\varepsilon \alpha_{1} s+\alpha_{3} s+\alpha_{2}(1+\tau s)(\varepsilon+1)\right)+\alpha_{2} s(1+\tau s)\right] \\
a_{3}=\frac{s^{4} \alpha_{2}}{\alpha_{3}-1}\left(1+\tau_{0} s\right)(1+\tau s) .
\end{gathered}
$$

Equation (3.3) can be factorized as

$$
\left(\nabla^{2}-k_{1}^{2}\right)\left(\nabla^{2}-k_{2}^{2}\right)\left(\nabla^{2}-k_{3}^{2}\right)(\bar{e}, \bar{\theta}, \bar{C})=0,
$$

where $k_{1}, k_{2}$, and $k_{3}$ are the roots with positive real parts of the characteristic equation

$$
k^{6}-a_{1} k^{4}+a_{2} k^{2}-a_{3}=0
$$

The solution of (3.3), which is bounded as $r \rightarrow 0$, is given by

$$
\bar{\theta}(r, s)=\sum_{i=1}^{3} A_{i}(s) I_{0}\left(k_{i} r\right), \quad \bar{e}(r, s)=\sum_{i=1}^{3} A_{i}^{\prime}(s) I_{0}\left(k_{i} r\right), \quad \bar{C}(r, s)=\sum_{i=1}^{3} A_{i}^{\prime \prime}(s) I_{0}\left(k_{i} r\right),
$$

where the unknown functions $A_{i}(s), A_{i}^{\prime}(s)$, and $A_{i i}^{\prime \prime}(s)$ are to be determined below by using the boundary conditions in (2.22)-(2.24), and $I_{0}$ is the modified Bessel function of the 
first kind of order zero. Substituting from (3.7) into (3.2), we get

$$
\begin{gathered}
A^{\prime}{ }_{i}(s)=\frac{k_{i}^{2}\left[k_{i}^{2}-s\left(1+\tau_{0} s\right)\left(1-\varepsilon \alpha_{1}\right)\right]}{\varepsilon s\left(1+\tau_{0} s\right)\left[\left(\alpha_{1}+1\right) k_{i}^{2}-\alpha_{1} s^{2}\right]} A_{i}(s), \\
A^{\prime \prime}{ }_{i}(s)=\frac{k_{i}^{4}-k_{i}^{2}\left[s\left(1+\tau_{0} s\right)(1+\varepsilon)+\alpha s^{2}\right]+s^{3}\left(1+\tau_{0} s\right)}{\varepsilon s\left(1+\tau_{0} s\right)\left[\left(\alpha_{1}+1\right) k_{i}^{2}-\alpha_{1} s^{2}\right]} A_{i}(s) .
\end{gathered}
$$

We thus have

$$
\begin{gathered}
\bar{e}(r, s)=\sum_{i=1}^{3} \frac{k_{i}^{2}\left[k_{i}^{2}-s\left(1+\tau_{0} s\right)\left(1-\varepsilon \alpha_{1}\right)\right]}{\varepsilon s\left(1+\tau_{0} s\right)\left[\left(\alpha_{1}+1\right) k_{i}^{2}-\alpha_{1} s^{2}\right]} A_{i}(s) I_{0}\left(k_{i} r\right), \\
\bar{C}(r, s)=\sum_{i=1}^{3} \frac{k_{i}^{4}-k_{i}^{2}\left[s\left(1+\tau_{0} s\right)(1+\varepsilon)+s^{2}\right]+s^{3}\left(1+\tau_{0} s\right)}{\varepsilon s\left(1+\tau_{0} s\right)\left[\left(\alpha_{1}+1\right) k_{i}^{2}-\alpha_{1} s^{2}\right]} A_{i}(s) I_{0}\left(k_{i} r\right) .
\end{gathered}
$$

In the Laplace transform domain, from (2.7) and (3.9), we get

$$
\bar{u}(r, s)=\sum_{i=1}^{3} \frac{k_{i}\left[k_{i}^{2}-s\left(1+\tau_{0} s\right)\left(1-\varepsilon \alpha_{1}\right)\right]}{\varepsilon s\left(1+\tau_{0} s\right)\left[\left(\alpha_{1}+1\right) k_{i}^{2}-\alpha_{1} s^{2}\right]} A_{i}(s) I_{1}\left(k_{i} r\right)
$$

Thus, from (2.17), (2.20), (3.1), and (3.9)-(3.10) it can be obtained that

$$
\begin{aligned}
\bar{\sigma}_{r r}(r, s) & =\frac{1}{\beta^{2} \varepsilon s\left(1+\tau_{0} s\right)} \sum_{i=1}^{3} \frac{k_{i}^{2}-s\left(1+\tau_{0} s\right)\left(1-\varepsilon \alpha_{1}\right)}{\left(\alpha_{1}+1\right) k_{i}^{2}-\alpha_{1} s^{2}}\left(\beta^{2} s^{2} I_{0}\left(k_{i} r\right)-\frac{2 k_{i}}{r} I_{1}\left(k_{i} r\right)\right) A_{i}(s), \\
\bar{P}(r, s) & =\frac{\alpha_{2}(1+\tau s)}{\varepsilon\left(1+\tau_{0} s\right)} \sum_{i=1}^{3} \frac{k_{i}^{4}-k_{i}^{2}\left[s\left(1+\tau_{0} s\right)(1+\varepsilon)+\alpha s^{2}\right]+\alpha s^{3}\left(1+\tau_{0} s\right)}{k_{i}^{2}\left[\left(\alpha_{1}+1\right) k_{i}^{2}-\alpha \alpha_{1} s^{2}\right]} A_{i}(s) I_{0}\left(k_{i} r\right) .
\end{aligned}
$$

Making Laplace transform of both sides of boundary conditions (2.22)-(2.24), we thus arrive at the following set of linear equations:

$$
\begin{gathered}
\sum_{i=1}^{3} \frac{k_{i}^{2}-s\left(1+\tau_{0} s\right)\left(1-\varepsilon \alpha_{1}\right)}{\left(\alpha_{1}+1\right) k_{i}^{2}-\alpha_{1} s^{2}}\left(\beta^{2} s^{2} I_{0}\left(k_{i} a\right)-\frac{2 k_{i}}{a} I_{1}\left(k_{i} a\right)\right) A_{i}(s)=0, \\
\sum_{i=1}^{3} A_{i}(s) I_{0}\left(k_{i} a\right)=\frac{\theta_{0}}{s}, \\
\sum_{i=1}^{3} \frac{k_{i}^{4}-k_{i}^{2}\left[s\left(1+\tau_{0} s\right)(1+\varepsilon)+\alpha s^{2}\right]+\alpha s^{3}\left(1+\tau_{0} s\right)}{k_{i}^{2}\left[\left(\alpha_{1}+1\right) k_{i}^{2}-\alpha \alpha_{1} s^{2}\right]} A_{i}=\frac{P_{0}}{s} \frac{\varepsilon\left(1+\tau_{0} s\right)}{\alpha_{2}(1+\tau s)} .
\end{gathered}
$$

Solving the linear system of (3.12) we can obtain the parameters $A_{1}, A_{2}$, and $A_{3}$. This completes the solution of the problem in the Laplace transform domain. 
Particular case. If we neglect the diffusion effect by eliminating (2.2) and (2.5), and by putting $\beta_{2}=c=0$ in (2.1) and (2.4), the expressions for temperature, displacement, and stress in a generalized thermoelastic medium are given by

$$
\begin{gathered}
\bar{\theta}(r, s)=\sum_{i=1}^{2} \frac{\varepsilon s\left(1+\tau_{0} s\right)}{k_{i}^{2}-s\left(1+\tau_{0} s\right)} A_{i}(s) I_{0}\left(k_{i} r\right), \quad \bar{u}(r, s)=\sum_{i=1}^{2} \frac{1}{k_{i}} A_{i}(s) I_{1}\left(k_{i} r\right), \\
\bar{\sigma}_{r r}(r, s)=\sum_{i=1}^{2}\left(\left[\beta^{2}-\frac{\varepsilon s\left(1+\tau_{0} s\right)}{k_{i}^{2}-s\left(1+\tau_{0} s\right)}\right] I_{0}\left(k_{i} r\right)-\frac{2}{k_{i} r} I_{1}\left(k_{i} r\right)\right) A_{i}(s),
\end{gathered}
$$

where

$$
\begin{gathered}
A_{i}(s)=(-1)^{i} \frac{f_{3-i} \theta_{0}}{s \Delta}, \quad i=1,2, \\
f_{i}=\left[\beta^{2}-\frac{\varepsilon s\left(1+\tau_{0} s\right) \lambda_{1}}{k_{i}^{2}-s\left(1+\tau_{0} s\right)}\right] I_{0}\left(k_{i} a\right)-\frac{2}{k_{i} a} I_{1}\left(k_{i} a\right), \\
\Delta=f_{1} n_{2}-f_{2} n_{1}, \quad n_{i}=\frac{\varepsilon s\left(1+\tau_{0} s\right)}{k_{i}^{2}-s\left(1+\tau_{0} s\right)} I_{0}\left(k_{i} a\right),
\end{gathered}
$$

where $k_{1}$ and $k_{2}$ are the roots with positive real parts of the characteristic equation

$$
k^{4}-k^{2}\left[s^{2}+s\left(1+\tau_{0} s\right)(1+\varepsilon)\right]+s^{3}\left(1+\tau_{0} s\right)=0 .
$$

\section{Inversion of the transforms}

We will now outline the numerical inversion method to obtain the solution of the problem in the physical domain. Following Honig and Hirdes [5], the Laplace transformed function can be inverted as follows:

$$
f(r, t)=\mathfrak{E}^{-1}[\bar{f}(r, s)]=\frac{1}{2 \pi \mathbf{i}} \int_{v-\mathbf{i} \infty}^{v+\mathbf{i} \infty} e^{s t} \bar{f}(r, s) d s .
$$

Let $s=v+i w(v, w \in \mathbb{R})$, the above formulation can be written as

$$
f(r, t)=\frac{e^{v t}}{2 \pi} \int_{-\infty}^{\infty} e^{\mathbf{i} w t} \bar{f}(r, v+i w) d w
$$

Expanding the function $h(r, t)=e^{-v t} f(r, t)$ in a Fourier series in the interval $[0,2 T]$, Durbin [3] derived the approximation formula

$$
\begin{aligned}
f(r, t)=\frac{e^{v t}}{T}[ & -\frac{1}{2} \mathfrak{R} e\{\bar{f}(r, v)\}+\sum_{k=0}^{\infty} \mathfrak{R} e\left[\bar{f}\left(r, v+\mathbf{i} \frac{k \pi}{T}\right)\right] \cos \left(\frac{k \pi}{T} t\right) \\
& \left.-\sum_{k=0}^{\infty} \mathfrak{J} m\left[\bar{f}\left(r, v+\mathbf{i} \frac{k \pi}{T}\right)\right] \sin \left(\frac{k \pi}{T} t\right)\right]-F_{1}(r, v, t, T),
\end{aligned}
$$


Table 5.1. Values of the constants.

\begin{tabular}{lll}
\hline$\alpha_{c}=1.98(10)^{-4} \mathrm{~m}^{3} / \mathrm{kg}$ & $\rho=8954 \mathrm{~kg} / \mathrm{m}^{3}$ & $\alpha_{t}=1.78(10)^{-5} \mathrm{~K}^{-1}$ \\
$c_{E}=383.1 \mathrm{~J} /(\mathrm{kg} \mathrm{K})$ & $\mu=3.86(10)^{10} \mathrm{~kg} /\left(\mathrm{m} \mathrm{s}^{2}\right)$ & $\lambda=7.76(10)^{10} \mathrm{~kg} /\left(\mathrm{m} \mathrm{s}^{2}\right)$ \\
$\rho=8954 \mathrm{~kg} / \mathrm{m}^{3}$ & $k=386 \mathrm{~W} /(\mathrm{m} \mathrm{K})$ & $D=0.85(10)^{-8} \mathrm{~kg} \mathrm{~s} / \mathrm{m}^{3}$ \\
$c=1.2(10)^{4} \mathrm{~m}^{2} /\left(\mathrm{K} \mathrm{s}^{2}\right)$ & $b=0.9(10)^{6} \mathrm{~m}^{5} /\left(\mathrm{kg} \mathrm{s}^{2}\right)$ & $\eta_{0}=8886.73 \mathrm{~s} / \mathrm{m}^{2}$ \\
$T_{0}=293 \mathrm{~K}$ & $\tau_{0}=0.02 \mathrm{~s}$ & $\tau=0.2 \mathrm{~s}$ \\
\hline
\end{tabular}

where $F_{1}(r, v, t, T)$ is the discretization error. It can be made arbitrarily small if the free parameter $v T$ is large (see [5]). As the infinite series in (4.3) can only be summed up to an infinite number $N$ of terms, hence the approximation value for $f(r, t)$ is

$$
\begin{aligned}
f_{N}(r, t)=\frac{e^{v t}}{T}[ & -\frac{1}{2} \mathfrak{R} e\{\bar{f}(r, v)\}+\sum_{k=0}^{N}\left[\mathfrak{R} e\left\{\bar{f}\left(r, v+\mathbf{i} \frac{k \pi}{T}\right)\right\} \cos \left(\frac{k \pi}{T} t\right)\right. \\
& \left.\left.-\mathfrak{J} m\left\{\bar{f}\left(r, v+\mathbf{i} \frac{k \pi}{T}\right)\right\} \sin \left(\frac{k \pi}{T} t\right)\right]\right] .
\end{aligned}
$$

Two methods are used to reduce the total error. First, the Korrecktur method is used to reduce the discretization error. Next, the $\varepsilon$-algorithm is used to reduce the truncation error and hence to accelerate convergence. The details can be seen in [5].

It should be noted that a good choice of the free parameters $N$ and $v T$ is not only important for the accuracy of the results but also for the application of the Korrecktur method and the methods for the acceleration of convergence. The values of $v$ and $T$ are chosen according to the criteria outlined in [5].

\section{Numerical results}

The copper material was used and chosen for purposes of numerical evaluations. The materials constants of the problem are thus given in Table 5.1 in SI units [18].

Using these values, it was found that

$$
\varepsilon=0.0168, \quad \beta^{2}=4, \quad \alpha_{1}=5.43, \quad \alpha_{2}=0.533, \quad \alpha_{3}=36.240 .
$$

It should be noted that a unit of nondimensional time corresponds to $6.5(10)^{-12} \mathrm{~s}$, while a unit of nondimensional length corresponds to $2.7(10)^{-8} \mathrm{~m}$.

The numerical technique outlined above was used to obtain the temperature, radial displacement, radial stress, and concentration as well as the chemical potential distributions inside the cylinder $(a=2)$. These distributions are shown in Figures 5.1-5.5, respectively. The computation was carried out for three values of time, namely, $t=0.1, t=0.15$, and $t=0.2$. For the sake of brevity some computational results are not being presented here.

Figures 5.1, 5.2(a), and 5.3(a) show the temperature $\left(\theta_{0}=1\right)$, radial displacement, and radial stress distributions, respectively. In these figures, the solid line represents the solution corresponding to using the generalized equation of heat conduction due to Lord and Shulman (LS theory: $\tau_{0}=0.02 \mathrm{~s}$ ), while the dotted line represents the solution corresponding to the usage of the classical coupled equation of heat conduction (CT theory: 


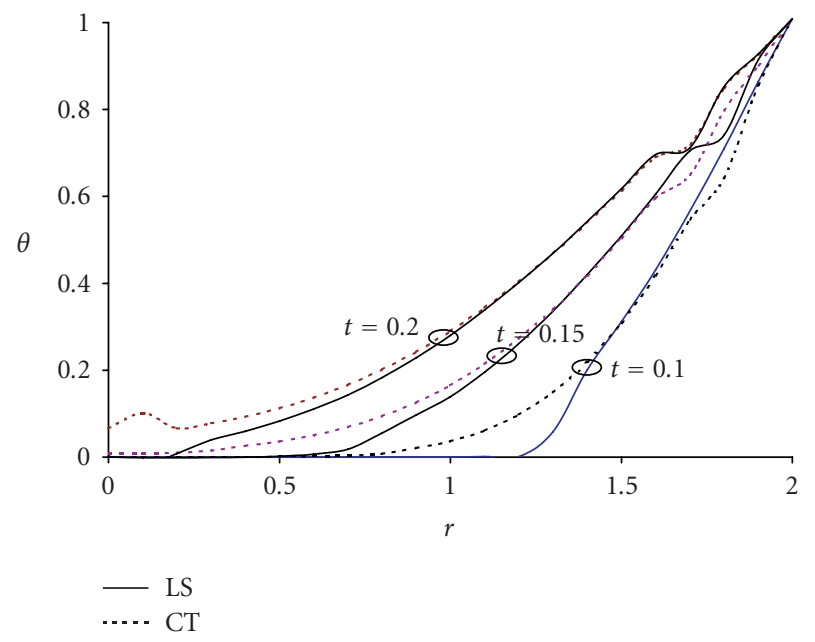

Figure 5.1. Temperature distribution.

$\tau_{0}=0$ ). It was found that near the surface of the cylinder where the boundary conditions dominate, the coupled and the generalized theories give very close results. Inside the cylinder, the solution is markedly different. This is due to the fact that thermal waves in the coupled theory travel with an infinite speed of propagation as opposed to a finite speed in the generalized case. In the coupled case, the solution fills the whole cylinder immediately. Thus, the solution is not identically zero (though it may be very small) for any small value of time. The solution obtained using the equations of generalized thermoelasticity, however, exhibits the behavior of finite speeds of wave propagation. For small values of time the solution is localized in a finite region of spaces surrounding the surface of the cylinder and is identically zero outside this region. This region grows with increasing time until it fills the hole cylinder. Its edge is the location of the wavefront. For large values of time, both theories give almost identical results. This is due to the short duration of the second sound effects.

Figure 5.2(b) shows that the medium adjacent to the cylinder surface undergoes expansion deformation because of thermal shock while the others undergo compressive deformation. The deformation is a dynamic process. With the passage of time, the expansion region moves insides gradually and becomes larger and larger. Thus the radial displacement becomes larger and larger. At a given instant, the nonzero region of radial displacement is finite, which is due to the wave effect of heat. It indicates that heat transfers into the deep of the medium with a finite velocity with the time passing. The more the considered instant, the more the thermal disturbed region and the radial displacement correspondingly.

In Figure 5.3(b), the radial stress at the cylinder surface is always zero, which agrees with the boundary condition prescribed. This coincided with the mechanical boundary condition that the cylinder surface is traction free. The medium close to the cylinder surface suffers from tensile stress which becomes larger with the time passing. The presence 


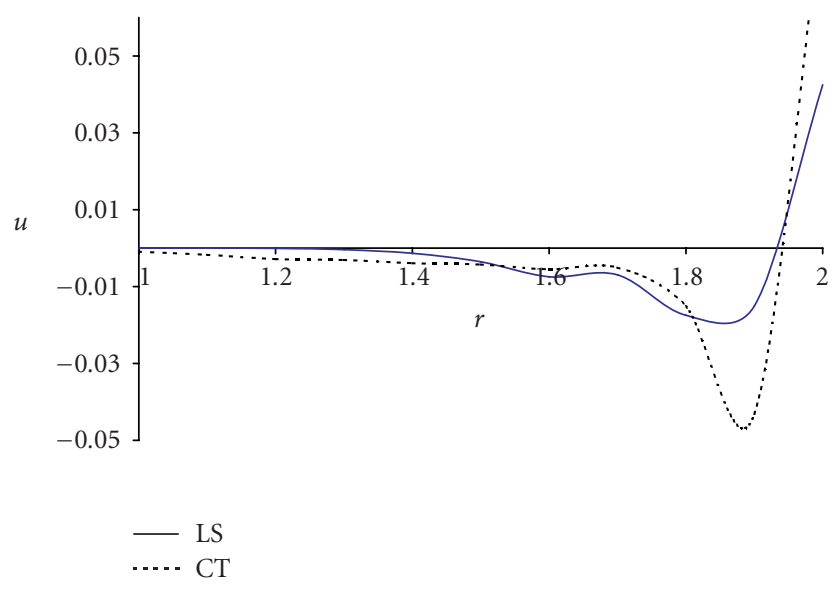

(a)

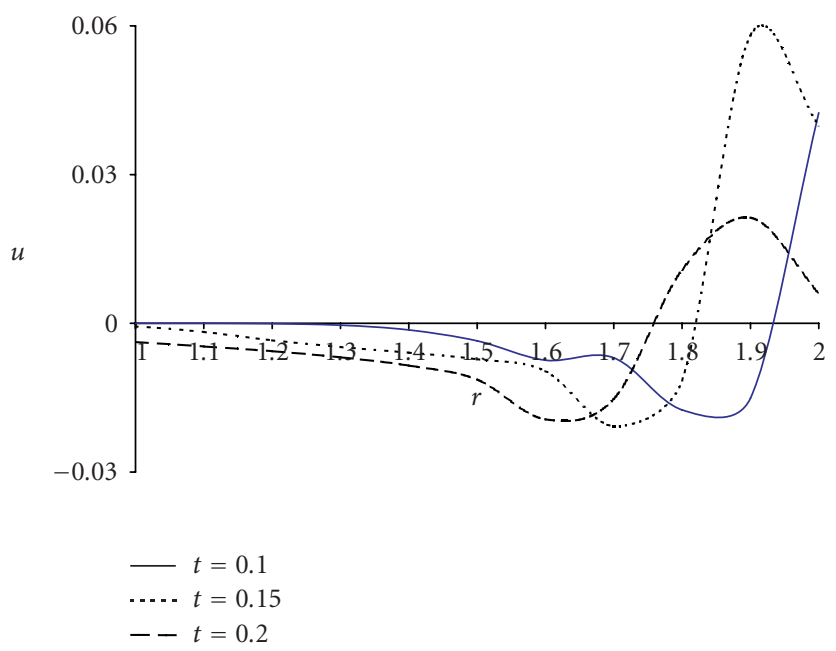

(b)

Figure 5.2. (a) Radial displacement distribution for $t=0.1$. (b) Radial displacement distribution.

of tensile stress close to the cylinder surface may be due to the influences of cross effects arising from the coupling of the fields of temperature, mass diffusion, and strain. Due to these cross effects, the thermal excitation results in that an additional mass concentration generates the additional field of thermal stresses.

Due to these cross effects described above, the concentration of the diffusive material is prominent in the medium close to the cylinder surface, as shown in Figure 5.4. The concentration increases to a maximum value at some distance from the cylinder surface and then gradually decrease to a zero value at $r=0$, which indicate that the equation satisfied by the concentration $C$ predicts finite speed of propagation. 
12 A generalized thermoelastic diffusion problem

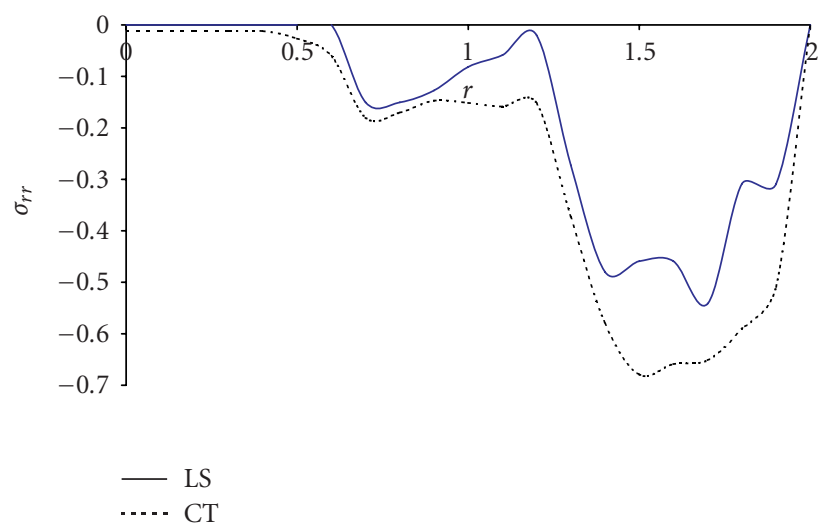

(a)

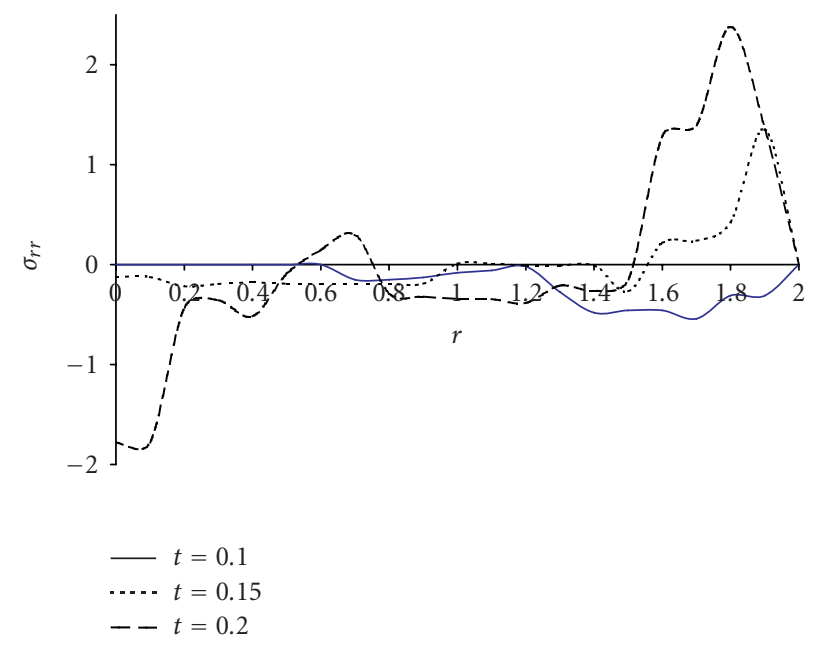

(b)

Figure 5.3. (a) Radial stress distribution for $t=0.1$. (b) Radial stress distribution.

Figure 5.5 shows the chemical potential distribution $\left(P_{0}=1\right)$. From the boundary of the cylinder surface, the chemical potential increases from unity value to a maximum value at some distance from the cylinder surface and then gradually decrease to a zero value at $r=0$, which confirm that the thermoelastic and diffusive waves propagate with finite speeds.

\section{Concluding remarks}

(1) Due to the complicated nature of the governing equations for the generalized thermoelastic diffusion theory, the work done in this field is unfortunately limited in number. The method used in this study provides a quite successfully approach in dealing with 


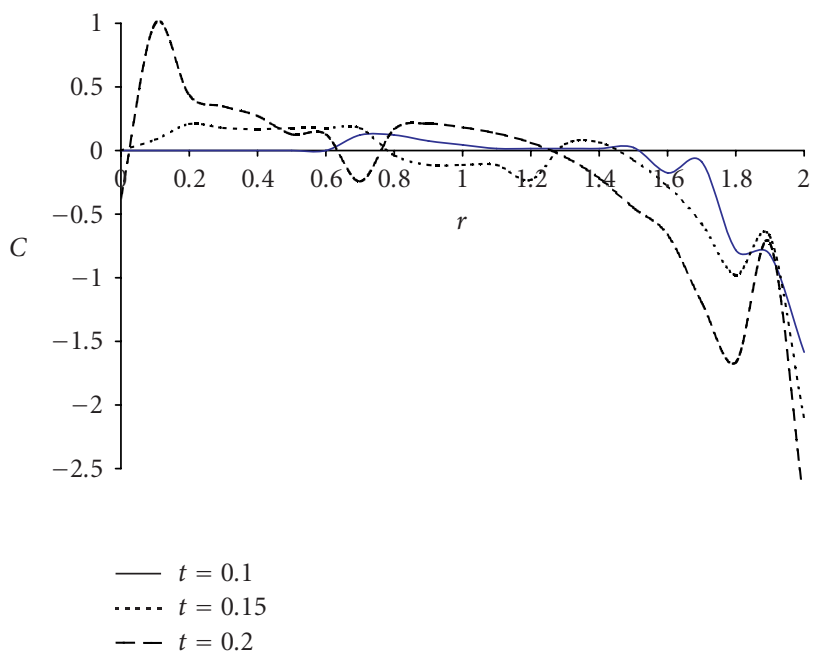

Figure 5.4. Concentration distribution.

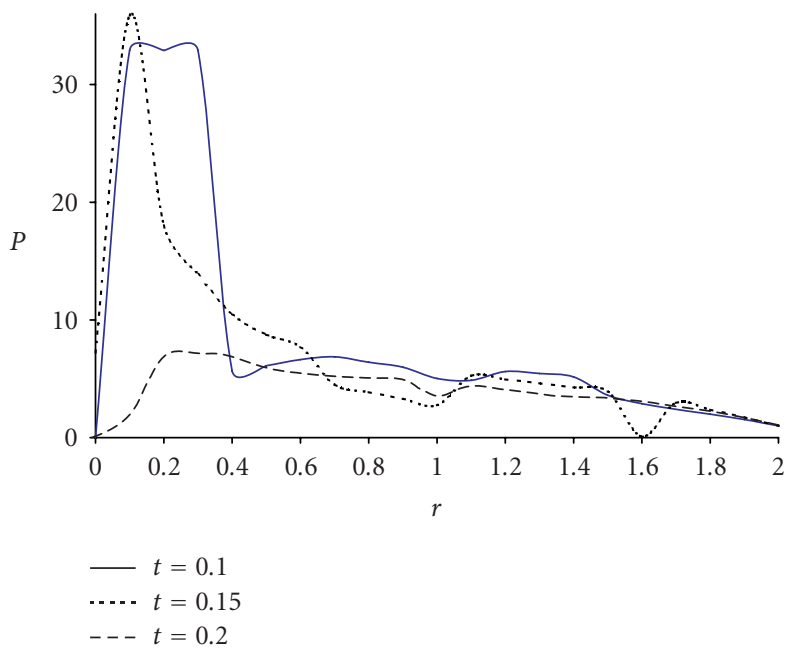

Figure 5.5. Chemical potential distribution.

such problems. This approach gives exact solutions in the Laplace domain without any assumed restrictions on the actual physical quantities that appear in the governing equations of the problem considered.

(2) The phenomenon of finite speeds of propagation is manifested in all theses figures. For the smallest values of time considered we see that the heat effects of the surrounding media are localized in a region adjacent to the walls. This region expands with the passage of time to fill the whole of the cylinder for the largest value of time. This region 
corresponds to the propagation of wavefronts from the surfaces of the cylinder. This is not the case for the coupled theory where thermal effects extends to the whole cylinder immediately.

(3) The results presented in this paper should prove useful for researchers in material science, designers of new materials, low-temperature physicists, as well as for those working on the development of a theory of hyperbolic thermodiffusion. Cross effects of heat and mass diffusion exchange with the environment arising from and inside nuclear reactors influence their design and operations [11]. Study of the phenomenon of diffusion is also used to improve the conditions of oil extractions (seeking ways of more efficiently recovering deposits) [17].

\section{Acknowledgment}

The author would like to thank the reviewers for their critical review and valuable comments which improved the paper thoroughly.

\section{References}

[1] M. Aouadi, Discontinuities in an axisymmetric generalized thermoelastic problem, International Journal of Mathematics and Mathematical Sciences 2005 (2005), no. 7, 1015-1029.

[2] _ Variable electrical and thermal conductivity in the theory of generalized thermoelastic diffusion, Zeitschrift für Angewandte Mathematik und Physik 57 (2006), no. 2, 350-366.

[3] F. Durbin, Numerical inversion of Laplace transforms: an efficient improvement to Dubner and Abate's method, The Computer Journal 17 (1974), 371-376.

[4] T. He, X. Tian, and Y. Shen, A generalized electromagneto-thermoelastic problem for an infinitely long solid cylinder, European Journal of Mechanics-A/Solids 24 (2005), no. 2, 349-359.

[5] G. Honig and U. Hirdes, A method for the numerical inversion of Laplace transforms, Journal of Computational and Applied Mathematics 10 (1984), no. 1, 113-132.

[6] H. Lord and Y. Shulman, A generalized dynamical theory of thermoelasticity, Journal of the Mechanics and Physics of Solids 15 (1967), no. 5, 299-309.

[7] W. Nowacki, Dynamic problems of thermodiffusion in elastic solids, Proceedings of Vibration Problems 15 (1974), 105-128.

[8] Dynamical problem of thermodiffusion in solids. I, Bulletin de l'Académie Polonaise des Sciences. Série des Sciences Techniques 22 (1974), 55-64.

[9] __ Dynamical problem of thermodiffusion in solids. II, Bulletin de l'Académie Polonaise des Sciences. Série des Sciences Techniques 22 (1974), 205-211.

[10] Dynamical problem of thermodiffusion in solids. III, Bulletin de l'Académie Polonaise des Sciences. Série des Sciences Techniques 22 (1974), 257-266.

[11] J. L. Nowinski, Theory of Thermoelasticity with Applications, Sijthoff \& Noordhoff International, Alphen Aan Den Rijn, 1978.

[12] B. Santawa and S. K. Roychoudhuri, Magneto-thermo-elastic interactions in an infinite isotropic elastic cylinder subjected to aperiodic loading, International Journal of Engineering Science 35 (1997), no. 4, 437-444.

[13] H. H. Sherief, Problem in electromagneto thermoelasticity for infinitely long solid conducting circular cylinder with thermal relaxation, International Journal of Engineering Science 32 (1994), no. 7, 1137-1149.

[14] H. H. Sherief and M. A. Ezzat, A problem in generalized magneto-thermoelasticity for an infinitely long annular cylinder, Journal of Engineering Mathematics 34 (1998), no. 4, 387-402. 
[15] H. H. Sherief, F. A. Hamza, and H. Saleh, The theory of generalized thermoelastic diffusion, International Journal of Engineering Science 42 (2004), no. 5-6, 591-608.

[16] H. H. Sherief and H. Saleh, A half-space problem in the theory of generalized thermoelastic diffusion, International Journal of Solids and Structures 42 (2005), no. 15, 4484-4493.

[17] B. Singh, Reflection of $P$ and $S V$ waves from free surface of an elastic solid with generalized thermodiffusion, Journal of Earth System Science 114 (2005), no. 2, 159-168.

[18] L. Thomas, Fundamentals of Heat Transfer, Prentice-Hall, New Jersey, 1980.

Moncef Aouadi: Department of Mathematics and Computer Science, Rustaq Faculty of Education, P.O. Box 10, Rustaq 329, Sultanate of Oman

E-mail address: moncef_aouadi@yahoo.fr 


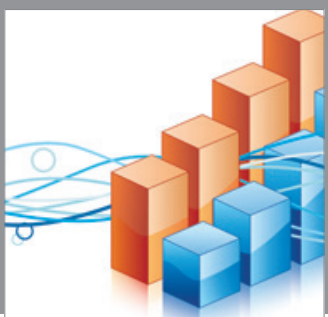

Advances in

Operations Research

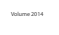

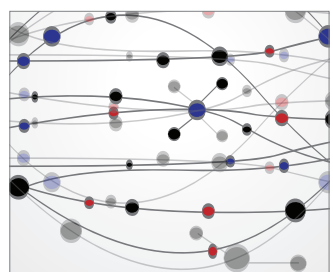

\section{The Scientific} World Journal
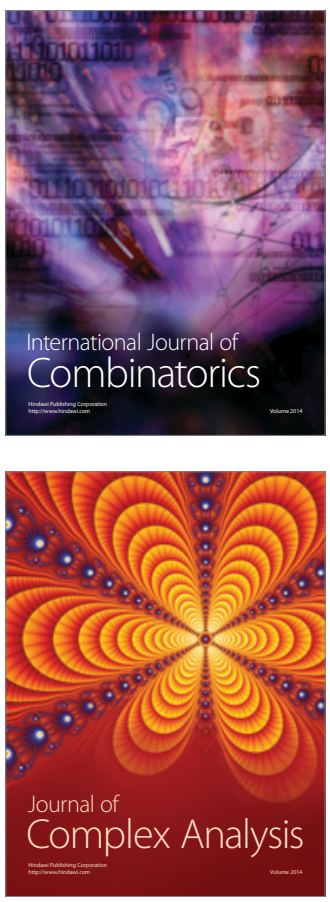

International Journal of

Mathematics and

Mathematical

Sciences
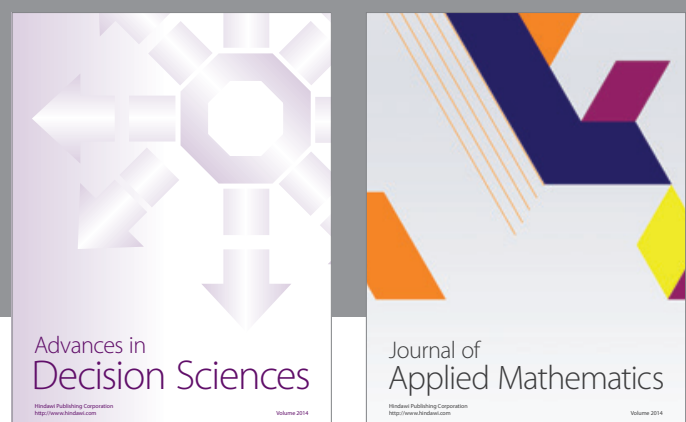

Journal of

Applied Mathematics
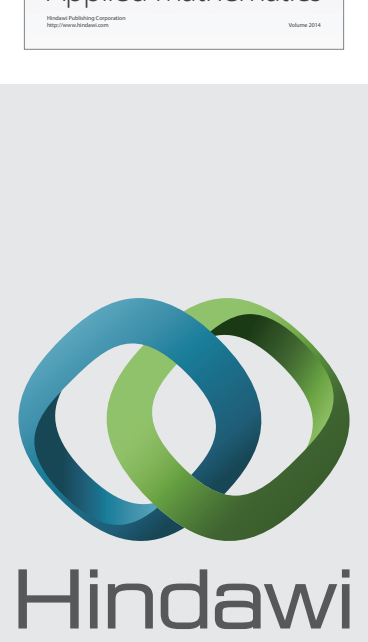

Submit your manuscripts at http://www.hindawi.com
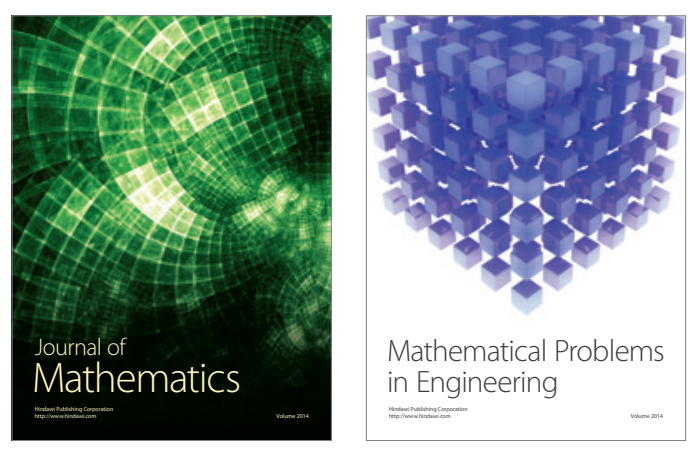

Mathematical Problems in Engineering
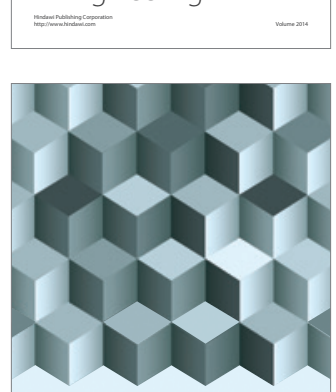

Journal of

Function Spaces
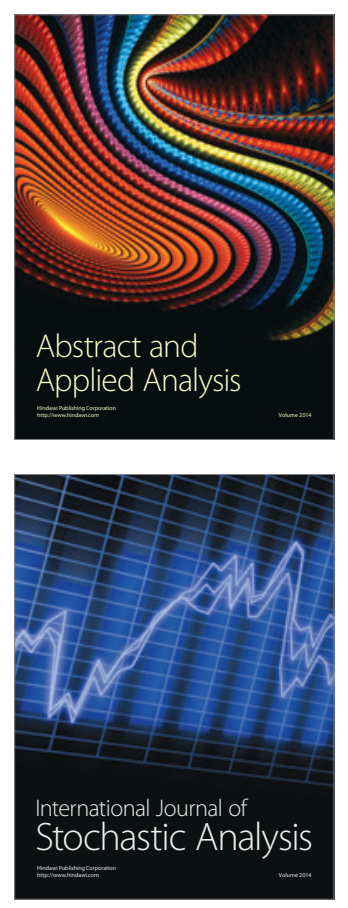

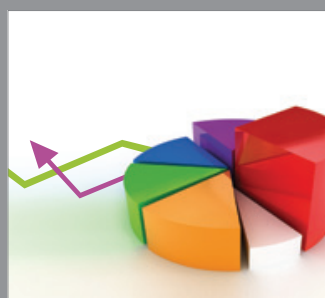

ournal of

Probability and Statistics

Promensencen
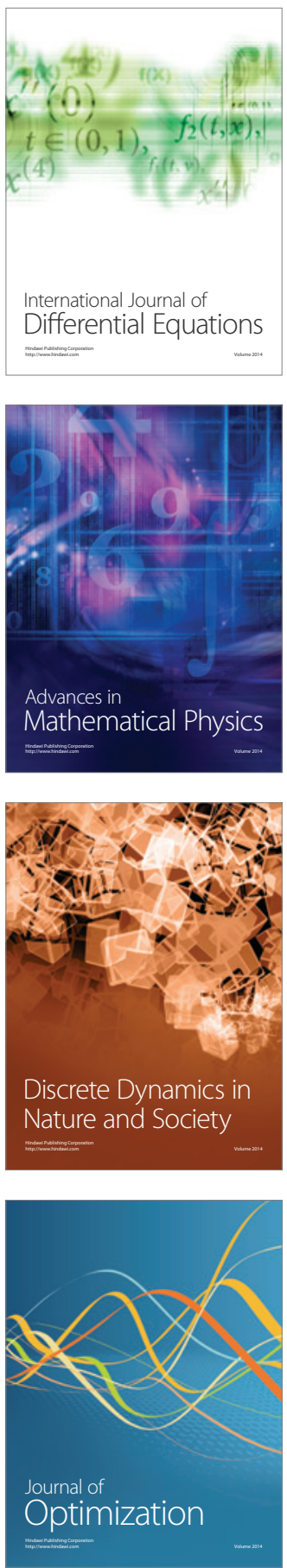